\title{
PI3K/Akt pathway-mediated HO-1 induction regulates mitochondrial quality control and attenuates endotoxin-induced acute lung injury
}

\author{
Jia Shi ${ }^{1} \cdot$ Jianbo Yu ${ }^{1,2} \cdot$ Yuan Zhang ${ }^{2} \cdot$ Lili $\mathrm{Wu}^{2} \cdot$ Shuan $\mathrm{Dong}^{2} \cdot$ Lina $\mathrm{Wu}^{2} \cdot$ Lingzhi $\mathrm{Wu}^{3} \cdot$ Shihan $\mathrm{Du}^{2}$ • \\ Yanfang Zhang ${ }^{2} \cdot$ Daqing $\mathrm{Ma}^{3}$
}

Received: 8 December 2018 / Revised: 26 April 2019 / Accepted: 24 May 2019 / Published online: 30 September 2019

(c) The Author(s), under exclusive licence to United States and Canadian Academy of Pathology 2019

\begin{abstract}
Sepsis-related acute lung injury (ALI) remains a major cause of mortality in critically ill patients and lacks specific therapy. Mitochondrial dysfunction is involved in the progression of septic lung injury. Mitochondrial dynamics, mitophagy, and biogenesis converge to constitute the assiduous quality control of mitochondria (MQC). Heme oxygenase-1 (HO-1) protects against sepsis-induced ALI through the modulation of mitochondrial dynamics. However, the causal relationship between HO-1 and the general processes of MQC, and their associated cellular pathways in sepsis-related ALI remain ill-defined. Herein, lipopolysaccharide (LPS)-induced ALI in Sprague-Dawley rats together with LPS-induced oxidative injury in RAW264.7 macrophages were used to investigate whether the PI3K/Akt pathway-mediated induction of HO-1 preserves MQC and alleviates septic lung injury. After pretreatment with hemin, a potent inducer of HO-1, LPS-induced cell apoptosis, enhanced mitochondrial fragmentation, and mitochondrial membrane potential damage were significantly reduced in macrophages. In rats, these effects were accompanied by a higher survival rate, less damage to lung tissue, a $28.5 \%$ elevation in lung mitochondria MnSOD activity, and a 39.2\% increase in respiratory control ratios. Concomitantly, HO-1 induction preserved the dynamic process of mitochondrial fusion/fission (Mfn2, OPA1, Drp1), promoted mitochondrial biogenesis (NRF1, PGC1 $\alpha$, Tfam), and facilitated the key mediators of mitochondrial mitophagy (Parkin, PINK1) at mRNA and protein levels. Notably, LY294002, a PI3K inhibitor, or knockdown of PI3K by small interfering RNA significantly suppressed Akt phosphorylation, attenuated HO-1 induction, and further reversed these beneficial effects evoked by hemin pretreatment in RAW264.7 cells or rats received LPS, indicating a direct involvement of PI3K/Akt pathway. Taken together, our results indicated that HO-1 activation, through PI3K/Akt pathway, plays a critical role in protecting lung from oxidative injury in the setting of sepsis by regulating MQC. HO-1 may therefore be a therapeutic target for the prevention sepsisrelated lung injury.
\end{abstract}

These authors contributed equally: Jia Shi, Jianbo Yu

Supplementary information The online version of this article (https:// doi.org/10.1038/s41374-019-0286-x) contains supplementary material, which is available to authorized users.

$\triangle$ Jianbo Yu

yujianbo11@126.com

1 Department of Anesthesiology, Tianjin Medical University NanKai Hospital, Tianjin, China

2 Department of Anesthesiology, Tianjin NanKai Hospital, Tianjin Medical University, Tianjin, China

3 Section of Anesthetics, Pain Medicine and Intensive Care, Department of Surgery and Cancer, Faculty of Medicine, Imperial College London, Chelsea and Westminster Hospital, London, UK

\section{Introduction}

Heme oxygenase-1 (HO-1) is cytoprotective as shown in various in vivo and in vitro models of cellular stress and organ injury [1,2]. During oxidative stress, induction of HO-1 exerts powerful antioxidant, anti-inflammatory, and anti-apoptotic properties, which are likely to be due to its degradation of heme and in turn, generation of cytoprotective byproducts such as ferrous iron, biliverdin, and carbon monoxide [3]. To date, the endogenous defensive role of the HO-1 system against sepsis-related lung injury (renamed as mild acute respiratory distress syndrome, ARDS) has been reported, which may be involved with the mitochondrial dynamics [4-6]. However, the overall regulatory mechanism at the subcellular level remains elusive. 
Mitochondria are not only known as the "energy powerhouse" of the cell but also mediate other cellular functions such as calcium management, cell growth, apoptosis, heme biosynthesis, inflammation, and oxygen sensing [7]. These organelles constitute a network of elongated filamentous structures through delicate cycles of fusion and fission, conducted by several prime dynamin-like GTPases including mitofusin (Mfn1/2), optic atrophy 1 (OPA1), and dynamin-related protein 1 (Drp1), which mediate mitochondrial quality control (MQC) in highly sophisticated ways [8]. It is vital to timely remove damaged or depolarized mitochondria and sustain adequate healthy mitochondria with intact membrane potential $(\Delta \Psi \mathrm{m})$ undergoing fission via the process of mitophagy, mediated uniquely by Parkin and PTEN-induced putative kinase 1 (PINK1) [9]. Healthy mitochondria are regenerated undergoing fusion via the process of biogenesis, that is regulated mainly by nuclear-encoded and mitochondria-encoded proteins, including nuclear respiratory factor (NRF1), peroxisome proliferator-activated receptor $\gamma$ coactivator 1 (PGC1 $\alpha$ ), and mitochondrial transcription factor A (Tfam) [10]. In sum, mitochondrial dynamics, mitophagy, and biogenesis converge to constitute the assiduous quality control of mitochondria, which play a crucial role in maintaining cellular homeostasis and functions [7,11]. Collectively, it is widely held that derangement in MQC evoked by massive oxidative stress is linked to the progression of sepsis-related multiple organ dysfunction [12-14]. Of note, there is an urgent need to exploit targeted therapies against sepsis by restoring mitochondrial homeostasis.

It is well known that the phosphatidylinositol-3-kinase (PI3K), a conserved family of signal transduction enzymes, is implicated in the regulation of cell growth, cycle entry, migration, and survival [15]. Protein kinase B (Akt) is activated via a PI3K pathway in which PI3K-mediated production of phosphatidylinositol-3,4,5-trisphosphate $\left(\mathrm{PIP}_{3}\right)$ leads to the recruitment of Akt to the cell membrane and then initiates Akt phosphorylation by phosphoinositidedependent kinase 1 (PDK-1) [16]. Numerous of studies set forth activation of the PI3K/Akt pathway-facilitated HO-1 induction and then afforded anti-apoptotic or antiinflammatory effects in response to oxidative injury [17-19]. While our previous studies indicated that the HO-1 system preserved the balance of mitochondrial dynamics to sustain the integrity of mitochondrial function and suppressed sepsis-induced lung injury [5]. However, the causal relationship between the general processes of MQC and PI3K/Akt-mediated HO-1 induction in sepsis-related lung injury remains unknown. In this study, under the condition of classically clinical relevant acute lung injury (ALI) model of Escherichia coli lipopolysaccharide (LPS) exposure to Sprague-Dawley rats or RAW264.7 macrophages [20], we investigated our hypothesis that PI3K/Akt pathway-dependent HO-1 induction appeared to have a generally protective effects on MQC in the context of sepsis.

\section{Materials and methods}

\section{Reagents}

Dulbecco's modified Eagle's medium (DMEM), fetal bovine serum (FBS), and penicillin-streptomycin were purchased from Invitrogen (Grand Island, NY, USA). Antibodies against Akt (ab176657), phospho-Akt (phospho S473, ab81283), Nrf2 (ab137550), PGC1 $\alpha$ (ab54481), NRF1 (ab175932), Tfam (ab217313), Parkin (ab179812), and PINK1 (ab23707) were purchased from Abcam (Cambridge, MA). Anti-HO-1 (SC-10789), anti-Mfn2 (SC-50331), anti-OPA1 (SC-367890), anti-Drp1 (SC-32898), anti- $\beta$-actin, and horseradish peroxidase-labeled anti-rabbit $\operatorname{IgG}$ were from Santa Cruz Biotechnology (Santa Cruz, CA, USA). LY294002 was obtained from Calbiochem (San Diego, CA). 3-(4, 5Dimethylthiazol-2-yl) 2,5-diphenyltetrazolium bromide (MTT) assay kit was from Roche Applied Science (Roche, IN). Mitochondrial membrane potential detection kit (JC-1) was supplied by the Nanjing Jiancheng Bioengineering Institute (Nanjing, China). All other chemicals, including LPS (Escherichia coli O111: B4, L2880) and hemin, were supplied by Sigma-Aldrich (St. Louis, MO, USA).

\section{Cell culture}

Murine RAW264.7 macrophages were obtained from the American Type Culture Collection (ATCC, Rockville, MD) and cultured in DMEM supplemented with 10\% heatinactivated FBS and $1 \%$ penicillin-streptomycin, followed by incubation at $37^{\circ} \mathrm{C}$ in $5 \% \quad \mathrm{CO}_{2} / 95 \%$ air humidified atmosphere. Cells at $70-80 \%$ confluence were split at a density of $2 \times 10^{5}$ cells $/ \mathrm{ml}$ in six-well plates. The cells were rinsed with fresh medium and incubated with $1 \mu \mathrm{g} / \mathrm{ml}$ LPS in the absence of presence of LY294002 $(10 \mu \mathrm{M})$ and hemin (oxidized heme, $20 \mu \mathrm{M}$ ), all of which were dissolved in DMSO (final concentration $<0.1 \%$ ) (Fig. 1a) [5, 21]. After $24 \mathrm{~h}$ of incubation in cultures, adherent cells were harvested and processed for further measurements.

\section{Cell viability}

To test the effects of various reagents with different concentrations, $2 \times 10^{5}$ cell/well were seeded in 96-well plates and incubated overnight. Cell viability (\%) was evaluated using an MTT assay, which was utilized to estimate total cellular metabolic activity based on the reduction of the compound to the formazan by mitochondrial dehydrogenases [22]. Ten microliters MTT ( $5 \mathrm{mg} / \mathrm{ml}$ in PBS, pH 7.6) was added to each 


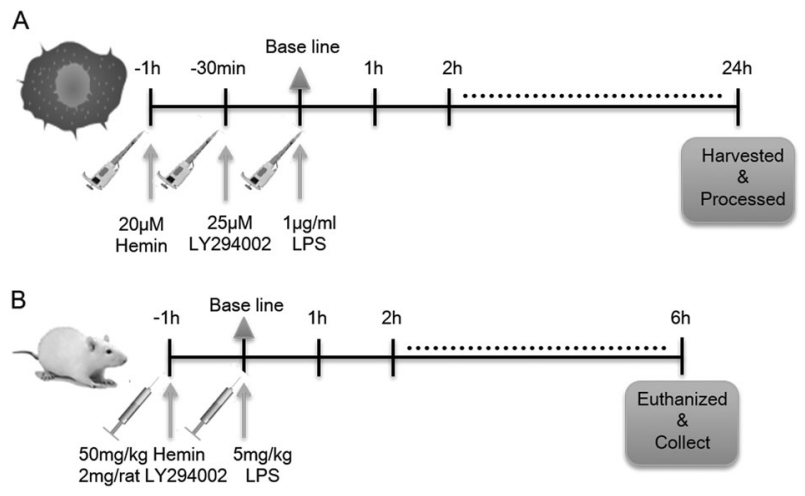

Fig. 1 Schematic diagram of the experimental protocols utilized in this study. a RAW264.7 macrophages were pre-treated with $20 \mu \mathrm{M}$ Hemin (a potent HO-1 inducer) for $1 \mathrm{~h}$ or $25 \mu \mathrm{M}$ LY294002 (a specific PI3K inhibitor) for $30 \mathrm{~min}$ ahead of $1 \mu \mathrm{g} / \mathrm{ml}$ LPS treatment and further incubated for $24 \mathrm{~h}$. b Sprague-Dawley rats were pre-treated intraperitoneally with $50 \mathrm{mg} / \mathrm{kg}$ hemin (dissolved in $50 \mu \mathrm{l} \mathrm{DMSO}$ ) or $2 \mathrm{mg} / \mathrm{rat}$ LY294002 (dissolved in $100 \mu \mathrm{l}$ DMSO) $1 \mathrm{~h}$ prior to LPS injection $(5 \mathrm{mg} / \mathrm{kg}$, caudal vein injection) and euthanized for tissue collection at $6 \mathrm{~h}$ after LPS administration. LPS lipopolysaccharide, HO-1 heme oxygenase-1, PI3K phosphatidylinositol-3-kinase

well at the end of each treatment and then incubated for $30 \mathrm{~min}$ at $37^{\circ} \mathrm{C}$. Optical density (OD) was measured at $570 \mathrm{~nm}$ with a microplate reader (Bio-Rad, Hercules, CA, USA).

\section{Mitochondrial membrane potential $(\Delta \Psi \mathrm{m})$}

The florescent, dual-emission mitochondrion-specific lipophilic, cationic dye JC-1 was used to assess the mitochondrial membrane potential $(\Delta \Psi \mathrm{m})$ according to the manufacturer's manuals. At the indicated times after sorts of experimental manipulations, macrophages were stained with $10 \mathrm{mg} / \mathrm{ml}$ 5,5',6,6'-tetrachloro-1,1', 3,3'-tetraethylbenzimidazolylcarbocyanine iodide (JC-1) for $20 \mathrm{~min}$ at $37^{\circ} \mathrm{C}$ in the dark [23]. Then, the cells were harvested by centrifugation $(1000 \times g, 5 \mathrm{~min})$, washed twice with phosphate-buffered saline (PBS), and resuspended in PBS. Immediately, they were analyzed by an FACSCalibur flow cytometer (BD Biosciences) using appropriate gate settings in FL1 (green) and FL2 (red) channels. Bright red fluorescence (excitation $490 \mathrm{~nm} /$ emission $590 \mathrm{~nm}$ ) was taken on by JC-1 aggregation in the mitochondrial matrix indicated high $\Delta \Psi \mathrm{m}$, while green fluorescence (excitation $488 \mathrm{~nm} / \mathrm{emis}-$ sion $530 \mathrm{~nm}$ ) was produced by JC-1 monomer in the cytosol indicated $\Delta \Psi \mathrm{m}$ collapse. The changes in the $\Delta \Psi \mathrm{m}$ were quantified by a bright red/green fluorescence ratio using FlowJo software (Tree Star Inc.).

\section{Transfection}

Negative control small interfering RNA (NC siRNA) and PI3K siRNA were designed at Shanghai GenePharma Co., Ltd (China). The sequences of siRNA (5-end prime; to 3-end prime) were as follows: PI3K siRNA: CCACCAG UCAAACCAUCAATT, NC SIRNA: UUCUCCGAACG UGUCACGUTT. RAW264.7 cells $\left(5 \times 10^{5} / \mathrm{ml}\right)$ were cultured in six-well plates and then transfected at $70-80 \%$ confluence with PI3K siRNA or NC siRNA using $5 \mu \mathrm{l}$ of Lipofectamine 2000 (No. 11668019; Invitrogen, USA) according to the manufacturer's protocols. The optimal transfection time was $24 \mathrm{~h}$ and then cells were stimulated with $1 \mu \mathrm{g} / \mathrm{ml}$ LPS with or without $20 \mu \mathrm{M}$ hemin. The efficiency of siRNA silencing was evaluated using western blot analysis. After incubation for $24 \mathrm{~h}$, the cells or the culture supernatants were collected for the subsequent detections.

\section{HO-1 enzyme activity}

The transfected RAW264.7 cells exposed to LPS in the absence or presence of hemin were harvested, added to the RIPA cell lysate, and the supernatant was collected. HO-1 enzyme activity was evaluated by monitoring the conversion of hemin into ferrous iron, carbon monoxide, and biliverdin by using HO-1 ELISA kits from the CUSABIO Biotechnology Co., Ltd (Catalog No. CSB-E08267r, Wuhan, China) based on the manufacturer's protocols [24]. Briefly, $200 \mu$ total reaction volume was added to each well. Then, the reduced type coenzyme II (NADPH) was added to initiate the reaction and incubated at $37{ }^{\circ} \mathrm{C}$ for $1 \mathrm{~h}$ in the dark. Immediately, $50 \mu \mathrm{l}$ stop solution was added to stop the reaction on ice. The formed bilirubin was extracted from $1 \mathrm{ml}$ of chloroform by vigorous vortexing three times for $10 \mathrm{~s}$ and measured using a spectrophotometer. Optical density at 464 and $530 \mathrm{~nm}$ of the organic phase was calculated and HO-1 enzyme activity was indicated as nanomoles of bilirubin formed per milligram of protein per hour.

\section{Western blotting}

Cells were washed twice in PBS, harvested by scraping, and resuspended in lysis buffer containing protease inhibitors and phosphatase inhibitors, as Hull et al. described [25]. A cell fractionation kit (Thermo Fisher Scientific, Rockford, IL) was used to separate mitochondrial cytoplasmic fractionation. Protein levels were quantified by a bicinchoninic acid (BCA) assay (Sigma, St. Louis, MO). Equal amounts of protein were subjected to $15 \%$ SDS-PAGE, transferred to a PVDF membrane (Bio-Rad, USA), and blocked with $4 \%$ nonfat dry milk in TBST (Sigma-Aldrich). Respectively, western blotting analysis for HO-1 (1:800), Nrf2(1:800), Akt (1:500), phospho-Akt (1:1000), NRF1 (1:1000), PGC1 $\alpha$ (1:500), Tfam (1:1000), Mfn2 (1:800), OPA1 (1:500), Drp1 (1:800), Parkin (1:100), PINK1 (1:100), and $\beta$-actin $(1: 1000)$ were performed on cell as delineated previously $[5,26]$. Bands were detected with a horseradish peroxidase-conjugated secondary antibody (1:5000) by means of the enhanced 
chemiluminescence (ECL) reagent (Bio-Rad, USA). Protein density was quantified on digitized images from the middynamic range, and data were expressed relative to $\beta$-actin.

\section{Real-time RT-PCR}

Total RNA was extracted from cultured cells using the TRIzol reagent (Invitrogen, Carsland, USA), as indicated by the manufacturers, and quantified spectrophotometrically at $260 \mathrm{~nm}$. Five microliters RNA obtained from each sample was reverse transcribed to cDNA using a reverse transcriptase (Promega, USA). Real-time RT-PCR was performed using SYBR Green Master Mix on an ABI Prism 7000 sequencedetector system (Applied Biosystems, Foster City, CA). The amplification conditions were as follows: pre-degeneration of the PCR mix $\left(10 \mathrm{~min}, 95^{\circ} \mathrm{C}\right)$, followed by 40 thermal cycles consist of denaturation $\left(30 \mathrm{~s}, 95^{\circ} \mathrm{C}\right)$, annealing $\left(5 \mathrm{~s}, 95^{\circ} \mathrm{C}\right)$, and extension $\left(34 \mathrm{~s}, 60^{\circ} \mathrm{C}\right)$. Primer sequences and the length of fragments for HO-1, Mfn2, OPA1, Drp1, PINK1, Parkin, Tfam, NRF1, and PGC1 $\alpha$ are listed in Table 1 entitled as the PCR primer sequences and the length of fragments. The comparative threshold cycle $\left(C_{\mathrm{T}}\right)$ method of relative quantification was used to determine the fold change in expression as depicted by Schmittgen TD et al. [27]. $\beta$-Actin served as an internal loading control.

\section{Transmission electron microscopy}

Mitochondrial ultrastructure was analyzed from cultured cells accordingly. Briefly, cells were washed with PBS (PH 7.4) and harvested by scraping lightly. And then, the cells were fixed overnight in $2.5 \%$ glutaradehyde/2\% paraformaldehyde in $0.1 \mathrm{M}$ phosphate buffer at $4{ }^{\circ} \mathrm{C}$ and processed on the routine protocols as previously described [28]. Ultrathin sections $(1 \mu \mathrm{m})$ were cut with a Leica Ultracut E ultramicrotome (Leica, Vienna, Austria) and stained with uranyl acetate and lead citrate for contrast. Digital images were acquired using a JEM-1400Plus transmission electron microscopy (TEM) (JEOL, Ltd, Japan). Analysis of mitochondria morphology was performed with the Image $\mathrm{J}$ software in a blinded manner.

\section{Animal model}

After obtained the experimental protocols approval by the Institutional Animal Care and Use Committee of Tianjin Nankai Hospital (Tianjin, China), a total of 36 SpragueDawley rats (male, 2 months old, 160-185 g) supplied by the Laboratory Animal Center of the Nankai Clinical Institution of Tianjin Medical University (Tianjin, China) were free access to food and water ad libitum and acclimatized to a 12-h light-dark cycle, were randomized into six groups $(n=6$ per group): (1) normal control (saline), (2) LPS (5 mg/kg, dissolved in $1 \mathrm{~m} 0.9 \%$ sodium chloride, caudal vein injection), (3) LPS plus hemin (a powerful inducer of HO-1), (4) LPS plus LY294002 (a specific inhibitor of PI3K), (5) LPS plus hemin plus LY294002, (6) LPS plus DMSO. To mimic the model of ALI, rats were anesthetized and implemented as described previously $[5,6]$. Respectively, the indicated concentrations of hemin $(50 \mathrm{mg} / \mathrm{kg}$, dissolved in $50 \mu \mathrm{DMSO})$ or LY294002 ( $2 \mathrm{mg} / \mathrm{rat}$, dissolved in $100 \mu \mathrm{l}$ DMSO) were administrated intraperitoneally $1 \mathrm{~h}$ prior to LPS injection (Fig. 1b) [3, 29]. The solutions containing the equal volume of sterile normal saline or DMSO were used as a control vehicle. The survival of the rats was monitored after LPS challenged in each group and then, the rats were empirically euthanized for lung tissue collection at $6 \mathrm{~h}$ after LPS application.

\section{Lung histology}

The lung specimens harvested from different groups were fixed in $10 \%$ formaldehyde. Routinely, the tissues were dehydrated,
Table 1 The PCR primer sequences and the length of fragments

\begin{tabular}{llll}
\hline Genes & Forward primer & Reverse primer & Length(bp) \\
\hline HO-1 & GAATCGAGCAGAACCAGCCT & CTCAGCATTCTCGGCTTGGA & 135 \\
Mfn2 & ACTTCTCCTCTGTTCCAGTTGT & GTGCTTGAGAGGGGAAGCAT & 181 \\
OPA1 & ACCTTGCCAGTTTAGCTCCC & ACCTAACAAGAGAAGGGCCTC & 131 \\
Drp1 & GCCTCAGATCGTCGTAGTGG & TGCTTCAACTCCATTTTCTTCTCC & 187 \\
Tfam & ATGGGTGTTGGGAAGTCT & CTCTCCTGTTGGTGGCTC & 168 \\
NRF1 & TCAGGGATAGGGGTTCA & TCAACCAGTTCGGCATA & 157 \\
PGC1 $\alpha$ & AGTGAAGATGAAAGTGA & TTGAGAAAATAAGGATT & 141 \\
Parkin & CTGCGTGTGATTTTTGCC & AGGTGCTCTGGGGTTCGT & 154 \\
PINK1 & GACCTCAAGTCCGACAACA & TGCCACCACGCTCTACAC & 145 \\
$\beta$-actin & TGTGTCCGTCGTGGATCTGA & TTGCTGTTGAAGTCGCAGGAG & 149 \\
\hline
\end{tabular}

HO-1 heme oxygenase-1, Mfn2 mitofusin 2, OPA1 optic atrophy 1, Drp1 dynamin-related protein 1, Tfam mitochondrial transcription factor A, NRF1 nuclear respiratory factor $1, P G C 1 \alpha$ peroxisome proliferatoractivated receptor $\gamma$ coactivator 1 
embedded in paraffin, sliced into microsections $(4 \mu \mathrm{m})$, and subsequently stained with hematoxylin-eosin (H\&E), and visualized under a light microscope ( $\times 400$ magnification). Quantitatively, the histopathology of the lung injury was scored in detail based on the following features: alveolar septa edema, airway congestion, interstitium widen or hyaline membrane formation, and neutrophil margination or infiltration [30]. Ten different visual fields were observed from each slice and the average score was calculated as preliminary study [5, 6]. Grading was performed by a pathologist who was blinded to the different groups.

\section{Mitochondria isolation and purification}

For mitochondria isolation, the harvested lung tissues were immersed in $4{ }^{\circ} \mathrm{C}$ PBS, minced with scissors, and followed by dispersion in Buffer A contained $0.225 \mathrm{~mol} / \mathrm{l}$ mannitol, $0.005 \mathrm{~mol} / \mathrm{l} \mathrm{MOPS}, 0.075 \mathrm{mr}$ sucrose, $0.002 \mathrm{~mol} / 1$ ethylenediaminetetraacetic acid (EDTA), and $1 \mathrm{mg} / \mathrm{ml}$ bovine serum albumin ( $\mathrm{pH}$ 7.4) using a Potter homogenizer via three passed at $300 \times g$, as delineated by Sommer et al. [31]. The $10 \%$ homogenate was centrifuged for $10 \mathrm{~min}$ at $3000 \times g$ at $4{ }^{\circ} \mathrm{C}$. And then, the supernatant was centrifuged at $10400 \times g$ for $10 \mathrm{~min}$ to obtain a crude mitochondrial filtrate. The pellet was resuspended in $1.0 \mathrm{ml}$ cold buffer B containing $0.225 \mathrm{~mol} / \mathrm{l}$ mannitol, $0.075 \mathrm{~mol} / \mathrm{l}$ sucrose, and $20 \mathrm{mmol} / \mathrm{l} \mathrm{Tris} / \mathrm{HCl}$ (pH 7.4) to serve as the mitochondrial fraction. Protein content was determined using the Pierce bichinonic acid (BCA) assay (Thermo Fisher Scientific, Schwerte, Germany).

\section{Mitochondrial respiratory function}

Mitochondrial oxygen consumption was assessed according to the protocols of Jacoby et al. [32], with slight modifications using a Clark-type oxygen electrode (Hansatech, United Kingdom). Briefly, rat lung mitochondria $(0.5 \mathrm{mg} / \mathrm{ml})$ in a buffer of $105 \mathrm{mM} \mathrm{KCl}, 5 \mathrm{mM} \mathrm{KH}{ }_{2} \mathrm{PO}_{4}$, $20 \mathrm{mM}$ Tris- $\mathrm{HCl}, 1 \mathrm{mM}$ diethylenetriaminepentaacetic acid, and $1 \mathrm{mg} / \mathrm{ml}$ fatty acid-free bovine serum albumin $(\mathrm{pH}$ 7.4) were incubated in the chamber for $10 \mathrm{~min}$ at $25^{\circ} \mathrm{C}$. The reaction was initiated with the substrates $5 \mathrm{mM}$ glutamate/10 $\mathrm{mM}$ succinate (state 4 , ST4), and the addition of $0.2 \mathrm{mM}$ ADP (state 3, ST3). The respiratory control ratio (RCR) was derived from the ratio of ST3/ST4 respiration.

\section{Manganese superoxide dismutase activity}

The enzymatic activity of manganese superoxide dismutase (MnSOD) in lung mitochondria of rats were determined by using Xanthine Oxidase assay kits from the Nanjing Jiancheng Bioengineering Institute (Nanjing, China) complied with the manufacturer' instructions. The activity of MnSOD was expressed as units per milligram protein ( $\mathrm{U} / \mathrm{mg}$ protein).

\section{Statistical analysis}

All values were obtained from at least five independent experiments for each condition in the current study. Of note, the sample sizes were estimated based on our previous studies in which $\mathrm{HO}-1 / \mathrm{CO}$ maintained mitochondrial dynamics to relieve septic ALI rather than a priori statistical power calculation [5]. Randomization methods were used to assign animals/cells to treatment groups and blinded assessments. Graph prism 5.0 (GraphPad Software, La Jolla, CA) was used for statistically analysis. Unless state otherwise, the distributions of continuous variables were expressed as mean $\pm \mathrm{SD}$. Significance of difference in mean values was detected by one-way or two-way analysis of variance (ANOVA) followed by Bonferroni's post hoc comparison tests. The lung injury scores were analyzed by nonparametric Mann-Whitmey $U$ test. The null hypothesis was rejected for $P<0.05$ with the two-tailed test. No experimental data were missing or lost in the statistical analysis.

\section{Results}

\section{H0-1 protected the lung from LPS-driven mitochondrial oxidative damage}

Septic lung injury is devastating clinical condition with a high mortality rate of up to $46 \%$, which is partially attributed to dysregulation of mitochondrial homeostasis initiated by remarkable oxidative stress [33]. As shown in Fig. 1, the role of HO-1 in regulating MQC has been currently investigated with a well-established model of endotoxin-induced lung injury in vitro and in vivo. To assess the impact of HO1 on LPS-mediated cell death, RAW264.7 cells were preincubated with $20 \mu \mathrm{M}$ hemin for $1 \mathrm{~h}$ or $25 \mu \mathrm{M}$ LY294002 for $30 \mathrm{~min}$ before $1 \mu \mathrm{g} / \mathrm{ml}$ LPS treatment up to $24 \mathrm{~h}$, as evaluated by an MTT assay (Fig. 2a). It was showed that $54.3 \pm 6.9 \%$ loss of cell viability subjected to LPS was further reduced to $38 \pm 3.9 \%$ by LY294002, known as a specific inhibitor of PI3K. Inversely, the addition of hemin, a potent inducer of HO-1, apparently increased the viability of cells to $76.7 \pm 9.3 \%(P=0.002)$. While no losses of cell viability were observed in RAW264.7 cells incubated with only hemin or LY294002 $(P>0.05)$. Of note, the vehicle, dimethyl sulfoxide (DMSO) (final concentration $<0.1 \%$ ), has been proven to be non-toxic to cells. To evaluate the effect of HO-1 on the survival rate of LPS-induced ALI in rats, the rats were monitored after intraperitoneally challenged with $50 \mathrm{mg} / \mathrm{kg}$ hemin or $2 \mathrm{mg}$ LY294002 $1 \mathrm{~h}$ prior to 
A

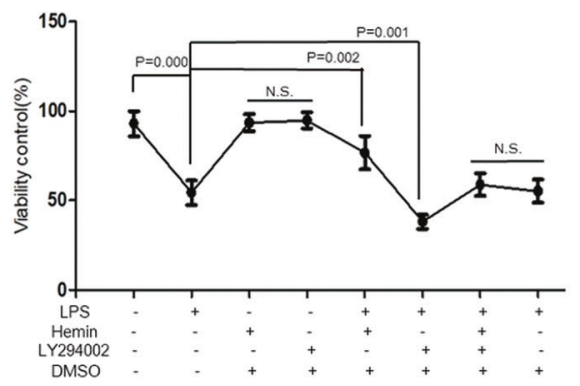

B

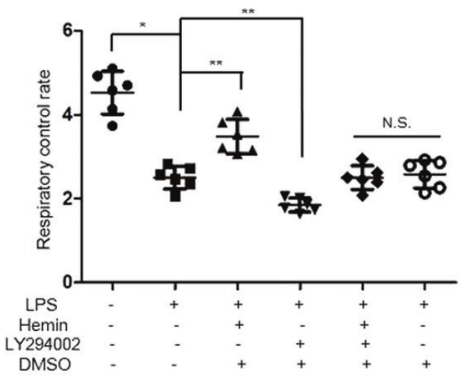

C

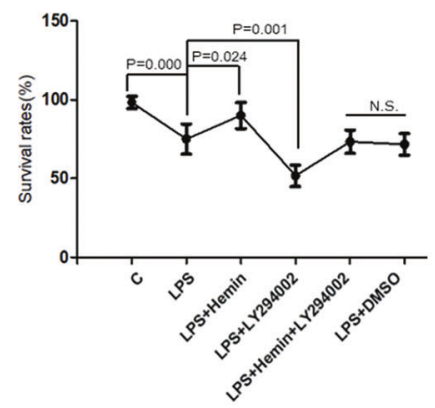

D

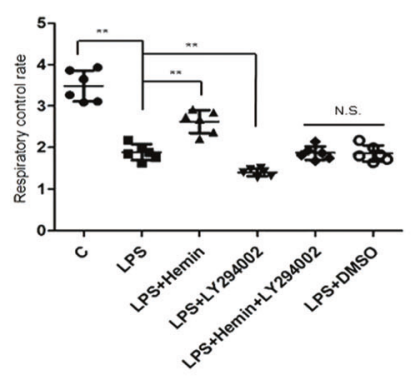

G

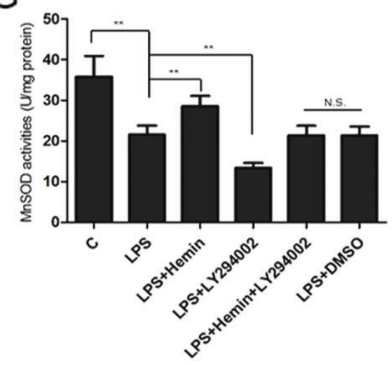

E

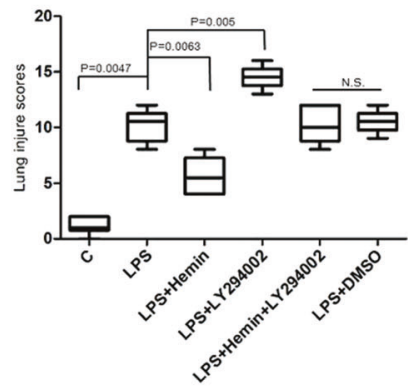

F
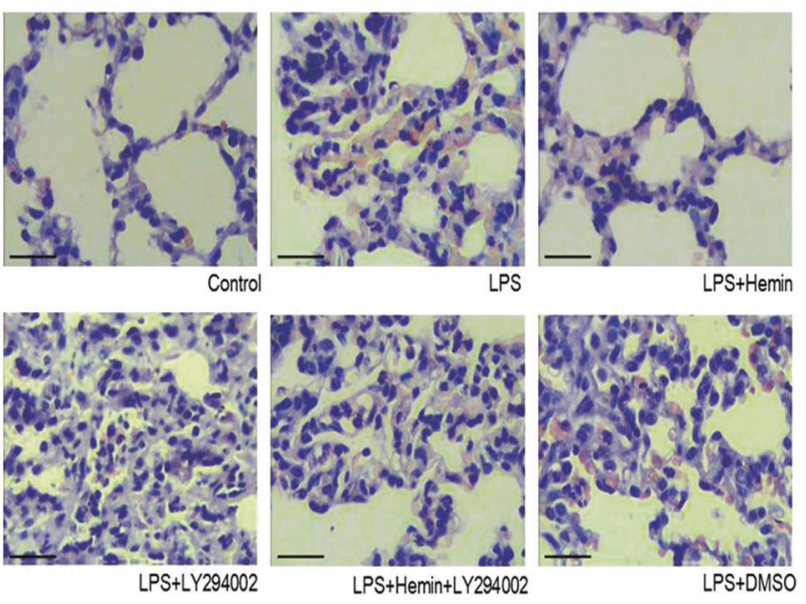

Fig. 2 Induction of HO-1 protected the lung from LPS-driven mitochondrial oxidative injury. a Cell viability at the indicated conditions was detected by MTT assay. b, d Respiratory control ratio (RCR) levels both in vitro and in vivo. $\mathbf{c}$ The survival rate of rats challenged by LPS with or without hemin or LY294002 treatment. Rats (6-10 animals per group) were monitored for lethality for up to $6 \mathrm{~h}$. e Semiquantification of lung tissues using lung injury scores. A five-point scale was applied to grade the degrees of lung injury based on the pathologic features of alveolar septa edema, airway congestion, interstitium widen or hyaline membrane formation, and neutrophil margination or infiltration: scores of $0=$ minimal damage, $1+=$ mild damage, $2+=$ moderate damage, $3+=$ severe damage, and $4+=$

LPS injection. As shown in Fig. 2c, the survival rate decreased to $75 \%$ at $6 \mathrm{~h}$ after LPS administration. Pretreatment with hemin significantly improved the survival rate of LPS-induced rats up to $90 \%$, which was abolished by the addition of LY294002 $(P<0.01)$. There was no significant difference between LPS plus DMSO group with LPS group, indicating that DMSO had no significant effects on the rats $(P>0.05)$.

To gain sight into the functional ability of mitochondrial oxidative phosphorylation, the RCR was detected (Fig. 2b, d). A significant decay of $44.37 \%$ in RCR levels were found maximal damage. Results are expressed as medians (range) using the Mann-Whitney $U$ test. f Photomicrographs of histopathologic changes of lung sections stained with hematoxylin and eosin (original magnification, $\times 400$ ). Scale bar: $100 \mu \mathrm{m}$. g MnSOD activity in lung mitochondria of rats. Data in a and $\mathbf{c}$ were analyzed using Mann-Whitney $U$ test followed by Bonferroni correction. Data in $\mathbf{b}, \mathbf{d}$, and $\mathbf{g}$ were presented as mean \pm s.d. using one-way ANOVA corrected with Bonferroni coefficient for multiple comparisons $(n=6$ replicates from three independent experiments). Significant differences were indicated with an asterisk: ${ }^{*} P<0.05,{ }^{* *} P<0.01,{ }^{* * *} P<0.001$. LPS lipopolysaccharide, RCR respiratory control ratio, MnSOD manganese superoxide dismutase, NS not significant

in RAW264.7 cells subjected to LPS, which were further depressed by LY294002, indirectly inhibited the induction of HO-1. As expected, this RCR-impaired effects of LPS were restored by the addition of hemin (LPS vs. LPS + hemin, $2.52 \pm 0.25$ vs. $3.48 \pm 0.37 ; P=0.001)$. Similar observations were recapitulated in the lung mitochondria of Sprague-Dawley rats after LPS stimulation.

Additionally, we utilized histological analysis by a light microscope to confirm the protective role of $\mathrm{HO}-1$ in attenuating septic lung injury of rats (Fig. 2e, f). Photomicrographs of lung slices revealed that hemin pretreatment 
ameliorated the histopathologic changes caused by LPS, as depicted by thickening of alveolar septum, infiltration of inflammatory cells, hemorrhage, and formation of hyaline membrane, which were aggravated by superposed with LY294002 (Fig. 2f). In support, semi-quantitative analysis of lung tissues by lung injury scores are summarized in Fig. 2e. The levels of lung injury scores were decreased in the pretreatment of hemin (5.5 [4-8]), while increased in the presence of LY294002 (14.5 [13-16]) in rats subjected to LPS (10.5 [8-11]). No such variables were observed in the lung of rats exposed to LPS and the addition of hemin plus LY294002 $(P>0.05)$.

As the key antioxidant enzyme in mitochondria, manganese superoxide dismutase (MnSOD) is one of the first in a chain of enzymes to protect mitochondria from oxidative damage by scavenging the superoxide anion [34]. Figure $2 \mathrm{~g}$ showed that, there was a significant reduction (about $39.82 \%$ of the control) in the enzymatic activity of MnSOD in the lung mitochondria of rats treated with $5 \mathrm{mg} / \mathrm{kg}$ LPS. Apparently, the levels of MnSOD were further decreased to $13.35 \pm 1.19 \%$ by pretreatment with LY294002, whereas the reduced MnSOD activities were significantly increased to $28.53 \pm 2.38 \%$ by HO-1 induction via hemin. Together, these results indicate that induction of HO-1 conferred protection against LPS-mediated mitochondrial damage and alleviated cellular oxidative injury or ALI of rats.

\section{PI3K/Akt pathway-mediated HO-1 induction promoted mitochondrial biogenesis}

As the nuclear transcription factor NRF1 and its coactivator PGC1 $\alpha$, as well as the mitochondria-specific transcription factor Tfam are major initiators of the mitochondrial biogenesis pathway, we determined the potential of HO-1 induction to promote mitochondrial biogenesis by real-time RT-PCR and western blot in vitro and in vivo. As illustrated in Fig. 3a, b, a distinct increase in the mRNA and protein levels of NRF1, PGC1 $\alpha$, and Tfam were detected in RAW264.7 macrophages elicited by LPS. Of note, expression of the mRNAs and proteins was upregulated up to 1.4-fold by induction of $\mathrm{HO}-1$ by pretreatment with hemin. In addition, to clarify whether the upstream kinase PI3K was involved in mitochondrial biogenesis, LY294002, a PI3K inhibitor, was pre-incubated in cells. Strikingly, this inhibitor negatively regulated the Akt phosphorylation, and the expression of HO-1, NRF1, PGC1 $\alpha$, and Tfam in both mRNA and protein levels $(P<0.01)$. Likewise, to evaluate the role of HO-1 in mitochondrial biogenesis in vivo, hemin or LY294002 was injected intraperitoneally in rats prior to LPS for $6 \mathrm{~h}$. In congruent with the results observed in macrophages, hemin pretreatment induced a dramatic increase in the levels of HO-1, phosphor-Akt, NRF1, PGC1 $\alpha$, and Tfam at mRNA and protein levels, whereas these effectors were reversely abrogated by cotreatment with LY294002 $(P<0.05)$ (Fig. 3c, d).

In light of the protective effect of PI3K/Akt pathwaymediated HO-1 induction extended to the subcellular levels, TEM was utilized to estimate the alterations in mitochondrial morphology, as shown in Fig. 4a, b. Quantitatively, there was a continued presence of swollen, fragmented mitochondria with disrupted cristae in LPS-stimulated macrophages, which exhibited marked mitochondrial damage characterized by loss of mitochondrial number, more swollen mitochondria contained abnormal electronlucent areas from LY294002 pre-treated cells. In contrast, altered mitochondrial architecture was restored nearly to normal by HO-1 induction with significant increases of mitochondrial number. Similar observations were also recapitulated in the rat lung tissues. Taken together, our data implied that lung protection by HO-1 induction in conjunction with the PI3K/Akt pathway facilitated mitochondrial biogenesis.

\section{PI3K/Akt pathway-mediated HO-1 induction preserved the mediators of mitochondrial dynamics}

In mammals, fusion of outer or inner mitochondrial membrane is mainly mediated by Mfn2 and OPA1, and mitochondrial fission requires the recruitment of Drp1 from cytosol to the outer mitochondrial membrane. Balance of mitochondrial fission/fusion encompassed mitochondrial dynamics has been suggested to serve as important quality control mechanism for preserving mitochondria [35]. LPS-induced cellular oxidative injury resulted in the fragmentation of damaged mitochondria, accompanied by lower levels of mitochondrial fusion gene and protein Mfn2 and OPA1, and higher levels of Drp1 mRNA and protein (Fig. 5a-e). In line with previous studies [5, 6], pretreatment with hemin ahead of LPS exhibited significant mitochondrial elongation, with a dramatic increase in the levels of mRNA and protein of HO-1, P-Akt, Mfn2, and OPA1, yet a distinct decrease of Drp1 mRNA and protein. However, mitochondria reverted back to a fragmented phenotype following preincubation with PI3K inhibitor LY294002, which deteriorated LPS-driven imbalance in mitochondrial dynamics. Importantly, these salutary effects of HO-1 induction were also evident in vivo.

Given that the fusion of mitochondrial inner membrane is dependent on intact mitochondrial membrane potential $(\Delta \Psi \mathrm{m})$ used as a hallmark of mitochondria functional status, we thus used JC-1 to ascertain the changes of $\Delta \Psi \mathrm{m}$. As showed in Fig. 5c, exposure to LPS of RAW264.7 cells led to a significant decline of $\Delta \Psi \mathrm{m}$, as compared with vehicletreated cells. Concordantly, pre-incubation with LY294002 caused a statistical loss of $\Delta \Psi \mathrm{m}$ in LPS-treated cells, along 


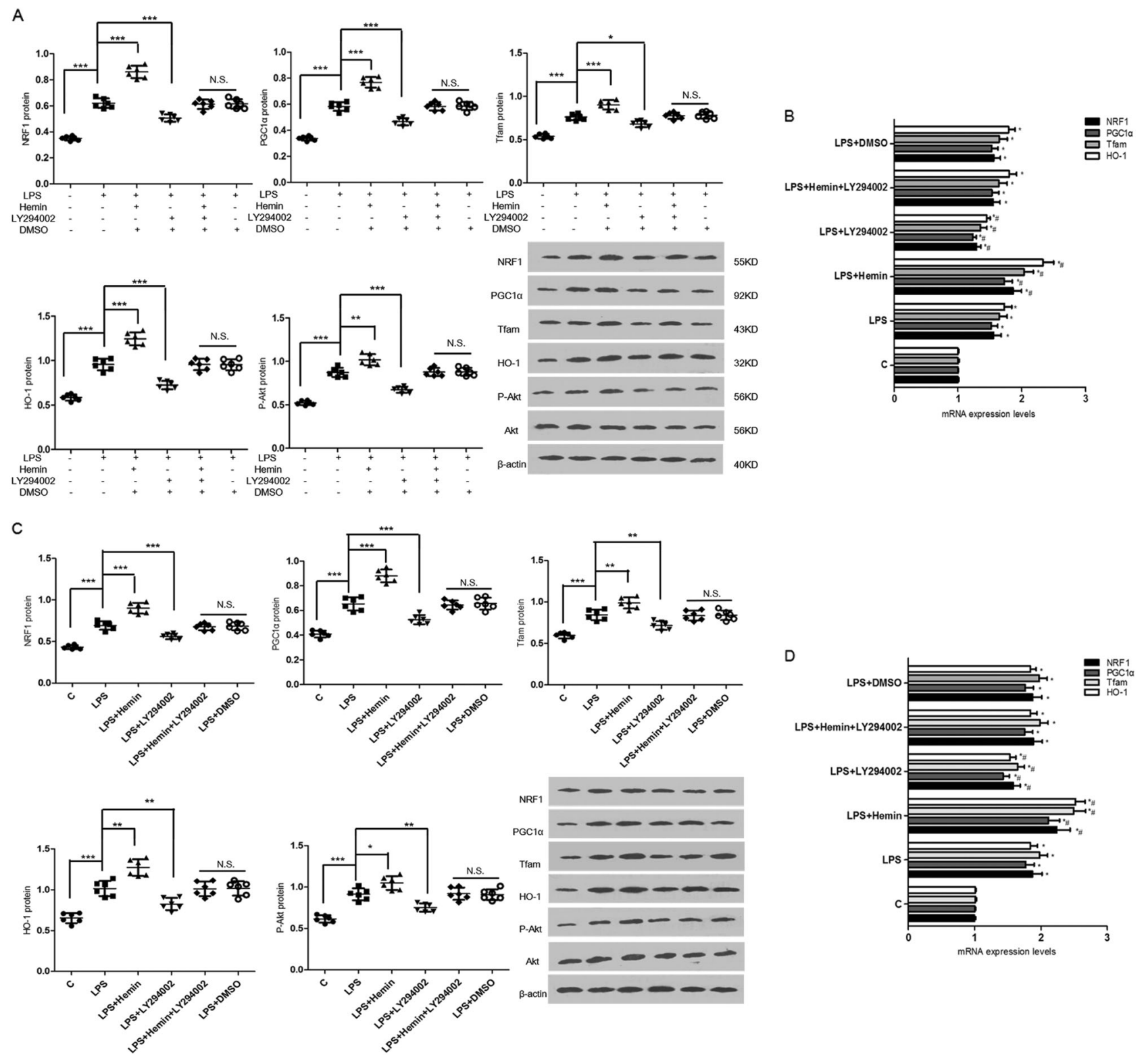

Fig. 3 Induction of HO-1 promoted mitochondrial biogenesis. a, b Example bands and quantification from western blots or real-time PCR to assess the expression of HO-1, phosphor-Akt, NRF1, PGC1 $\alpha$, and Tfam necessary for mitochondrial biogenesis in RAW264.2 cells. c, d The protein and the mRNA levels of HO-1, phosphor-Akt, NRF1, PGC1 $\alpha$, and Tfam in the lung tissues of rats. Band density was quantified relatively to $\beta$-actin. Data in Fig. a-d were expressed as mean \pm s.d., ${ }^{*} P<0.05$ vs. control and ${ }^{\#} P<0.05$ vs. LPS-treated

with severe fragmented mitochondria (LPS + LY294002 vs. LPS, $4.406 \pm 0.245$ vs. $9.531 \pm 0.622 ; P=0.000, n=6$ ). However, $82.7 \%$ elevation of the $\Delta \Psi \mathrm{m}$ manifested as the red-orange fluorescence intensity increased and the green fluorescence decreased, was observed in HO-1 induction during pretreatment with hemin. Collectively, these data suggested that PI3K/Akt pathway-mediated HO-1 induction shifted the balance toward a fusion phenotype, increased the $\Delta \Psi \mathrm{m}$, and preserved mitochondrial dynamics. vehicles. One-way ANOVA corrected with Bonferroni coefficient was used to detect statistically significant changes; $n=6$ per group. Significant differences were indicated with an asterisk: ${ }^{*} P<0.05,{ }^{* *} P<$ $0.01,{ }^{* * *} P<0.001$. LPS lipopolysaccharide, HO-1 heme oxygenase-1, Akt protein kinase $\mathrm{B}, \mathrm{NRF} 1$ nuclear respiratory factor 1 , PGC1 $\alpha$ peroxisome proliferator-activated receptor $\gamma$ coactivator 1 , Tfam mitochondrial transcription factor A, NS not significant

\section{PI3K/Akt pathway-mediated HO-1 induction prevented nonhomeostatic alterations of mitochondrial mitophagy}

Recent studies have reported that Parkin and PINK1 interacted directly to initiate mitochondrial mitophagy, which was an organelle-specific autophagy that facilitated the selective elimination of damaged or depolarized mitochondria undergoing fission [36]. Herein, we examined the 
A
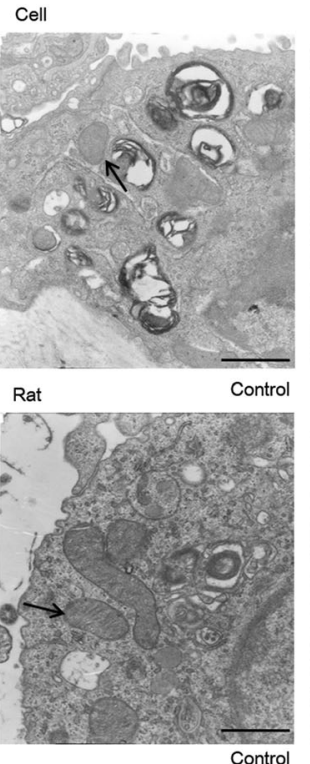

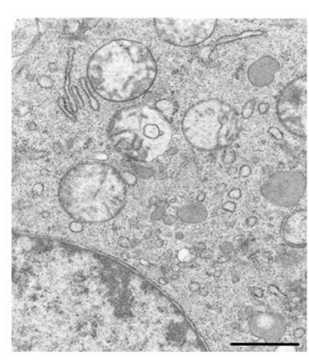

LPS

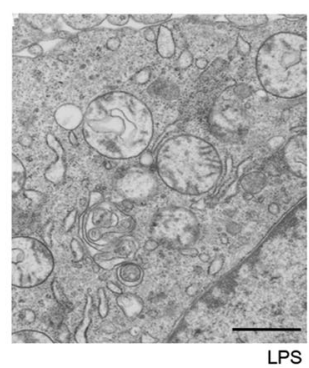

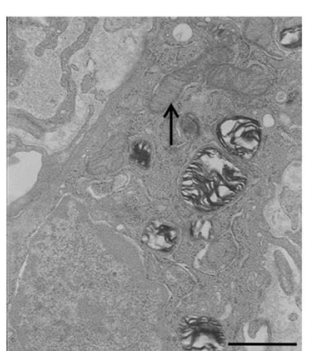

LPS+Hemin

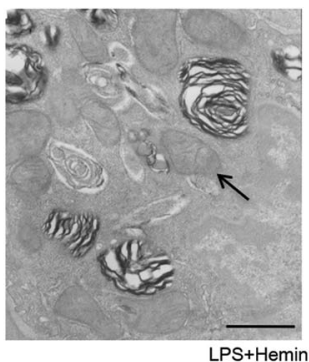

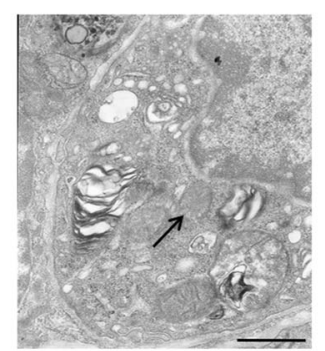

LPS+LY294002

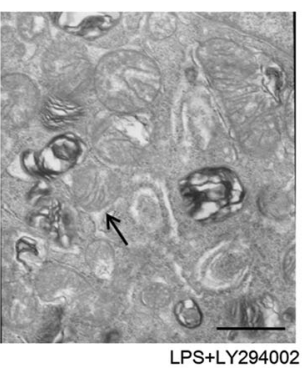

B

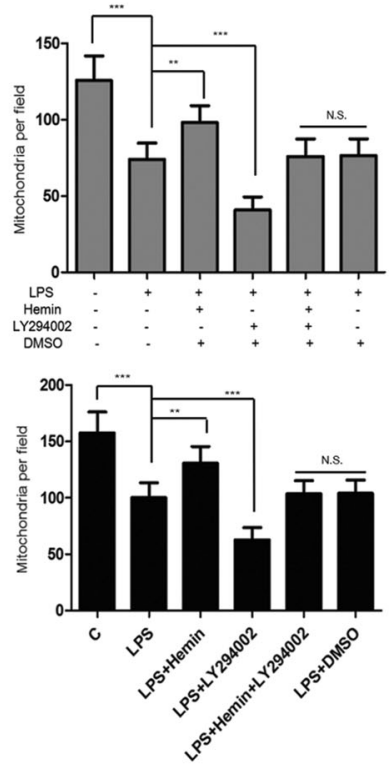

Fig. 4 The morphological alterations of mitochondria by transmission electron microscopy (TEM). a TEM images of mitochondria after treatment with LPS, hemin, or LY294002 in RAW264.7 cells or SD rats (original magnification, $\times 15,000$ ). Black arrows marked normal shape of mitochondria in the control group and damaged morphological mitochondria pre-induced by hemin or LY294002. Scale bar:
$2 \mu \mathrm{m}$. b The number of mitochondria from ten randomly selected fields per rat/cultured cell were quantitatively evaluated ( $n=6$ per group). Images were assessed by a blinded pathologist. Values were representative of mean \pm s.d., ${ }^{*} P<0.05,{ }^{* *} P<0.01,{ }^{* * *} P<0.001$, one-way ANOVA corrected with Bonferroni coefficient. LPS lipopolysaccharide, TEM transmission electron microscopy, NS not significant expression of Parkin and PINK1 at the transcriptional or translational levels at various experimental manipulations. As shown in Fig. 6a, b, higher mRNA and protein levels of both Parkin (48.83\% vs. 55.87\%) and PINK1 (53.12\% vs. $54.09 \%$ ) were observed in RAW264.7 cells exposed to LPS. Interestingly, the mRNA and protein levels of these mediators appeared to be increased substantially in pretreatment with hemin, as suggested by an increase in mitochondrial fusion as wells as enhanced P-Akt and HO-1 levels. However, LY294002 pre-incubation in cells counteracted hemin-induced alterations in the Parkin-PINK1 mitophagy pathway, which indicated a direct involvement of the PI3K/Akt pathway. To gain insight into the role of HO-1 in mitophagy susceptibility to LPS-induced septic lung injury in vivo, rats were pre-treated with hemin or LY294002, and RT-PCR or western blot analysis of Parkin and PINK1 were detected as well. As expected, HO-1 induction resulted in increased levels of Parkin and PINK1, concerned with prevailing mitochondrial fusion. In contrast, inclusion of LY294002 reversed the mRNA and protein expression of Parkin and PINK1 conferred by hemin pretreatment in LPS-exposed rats. Taken together, these results showed that PI3K/Akt pathway-mediated HO-1 induction prevented nonhomeostatic alterations of mitochondrial mitophagy, which is intimately linked to mitochondrial dynamics.

\section{PI3K/Akt/Nrf2 pathway participated in the regulation of HO-1-mediated MQC}

To further verify whether HO-1 regulated MQC through the activation of PI3K/Akt pathway, the PI3K siRNA and negative control siRNA were incubated in the RAW264.7 macrophages. As it was well known that the PI3K/Akt pathway mediated the activation of NF-E2-related factor-2 (Nrf2), which was involved in HO-1 induction [37]. Thus, we wondered whether Nrf2 played a role in the regulation of MQC. As shown in Fig. 7a-1, LPS exposure notably elevated the protein levels of NRF1, PGC1 $\alpha$, Tfam, Drp1, Parkin, PINK1, HO-1, phosphor-Akt, and Nrf2 nucleoprotein, and reduced the protein levels of Mfn2 and OPA1 compared with those in the control group $(P<0.01)$. Moreover, pretreatment of RAW264.7 cells with hemin evidently induced the expression levels of NRF1, PGC1 $\alpha$, Tfam, Mfn2, OPA1, Parkin, PINK1, HO-1, P-Akt protein and Nrf2 nucleoprotein, inhibited the expression level of Drp1 protein $(P<0.01)$. As expected, knockdown of PI3K by siRNA strongly blocked HO-1 induction-mediated maintenance of MQC balance, which presented lower protein levels of NRF1, PGC1 $\alpha$, Tfam, Mfn2, OPA1, Parkin, PINK1, HO-1, P-Akt protein, and Nrf2 nucleoprotein as well as higher protein levels of Drp1 $(P<0.01)$. Negative control siRNA did not significantly affect the 
A
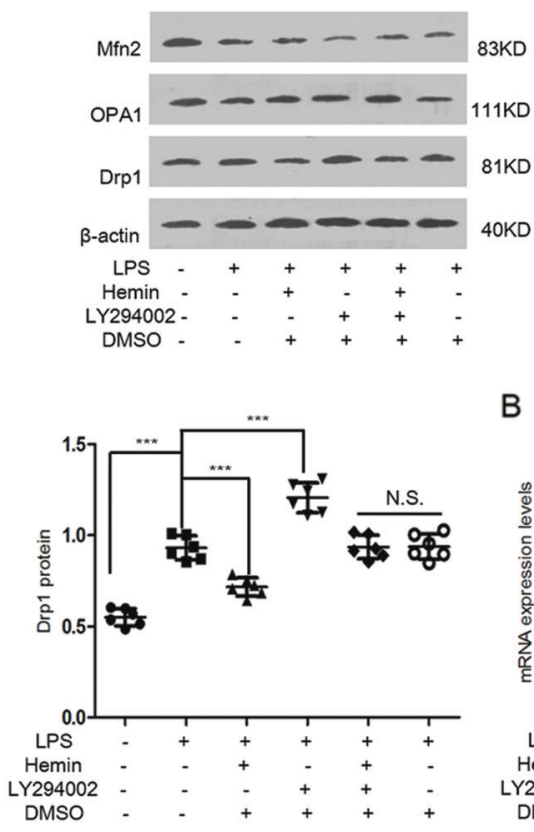

B
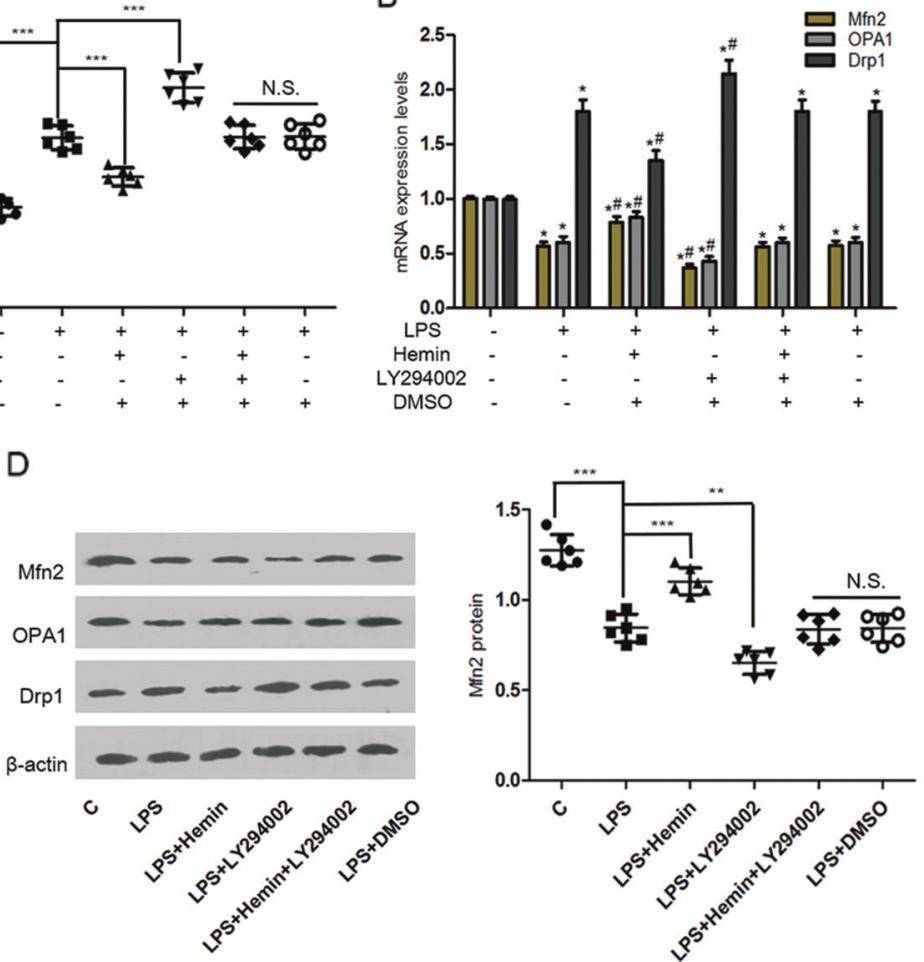

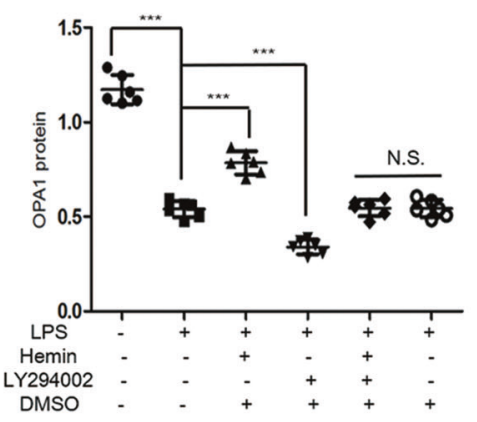

C

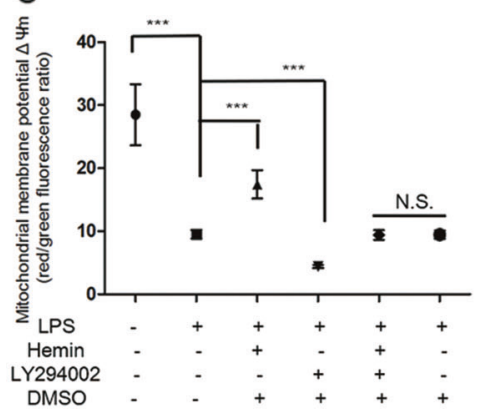

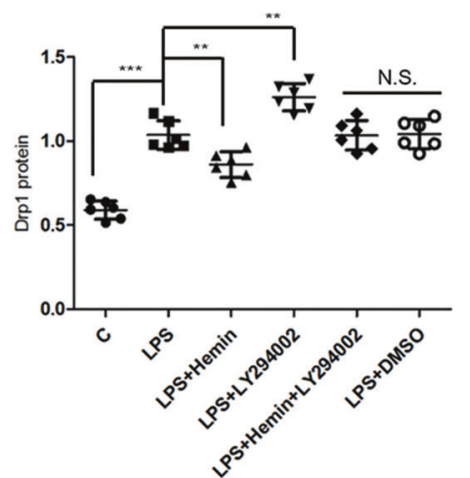

Fig. 5 Induction of HO-1 enhanced mitochondrial fusion whereas restrained fission. $\mathbf{a}, \mathbf{b}$ The expression of protein and mRNA of the dynamic markers including Mfn2, OPA1, and Drp1 for mitochondrial fusion/fission in RAW264.2 cells. c The mitochondrial membrane potential $(\Delta \Psi \mathrm{m})$ determined by JC-1 probe. Graphs represented as mean \pm s.d. of $n=6$ independent experiments. Significant differences were indicated with an asterisk: ${ }^{*} P<0.05,{ }^{* * *} P<0.01,{ }^{* * *} P<0.001$. d, e The levels of Mfn2, OPA1, and Drp1 mRNA and protein in the

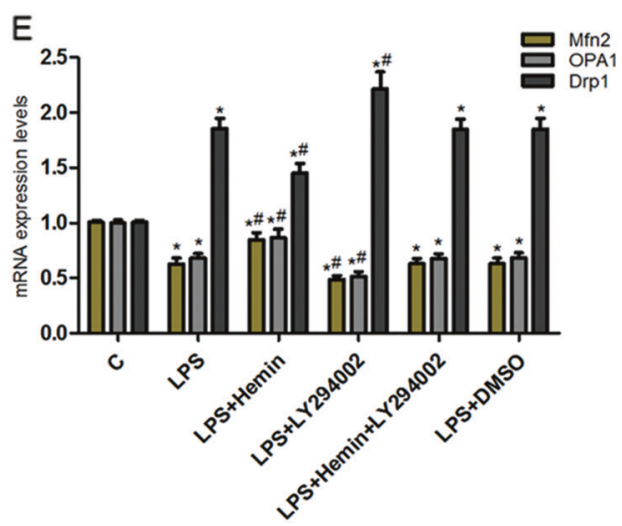

lung tissue of rats. $\beta$-Actin served as a standard for protein loading. Values were expressed as mean \pm s.d. of six individual samples using one-way ANOVA corrected with Bonferroni coefficient for multiple comparisons. "Significant difference from controls $(P<0.05)$. ${ }^{\#}$ Significant difference from LPS-treated cells or rats $(P<0.05)$. LPS lipopolysaccharide, HO-1 heme oxygenase-1, Mfn2 mitofusin 2, OPA1 optic atrophy 1 , Drp1 dynamin-related protein $1, \Delta \Psi \mathrm{m}$ mitochondrial membrane potential, NS not significant 
A

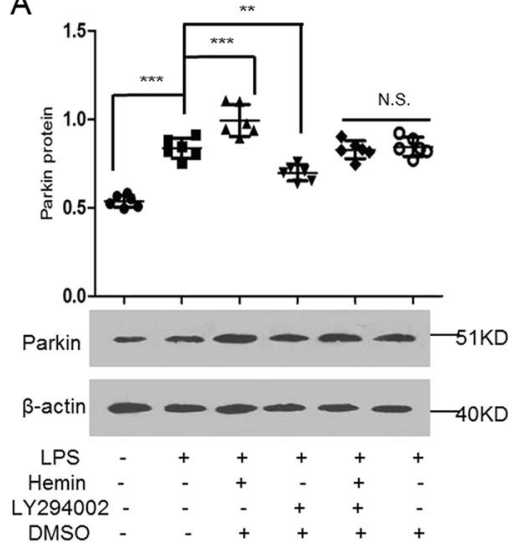

C
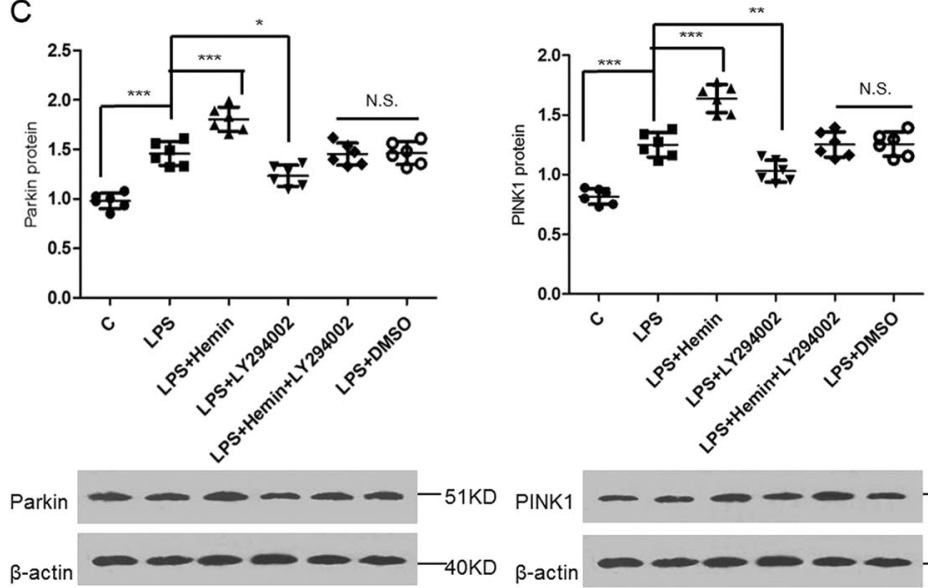

B
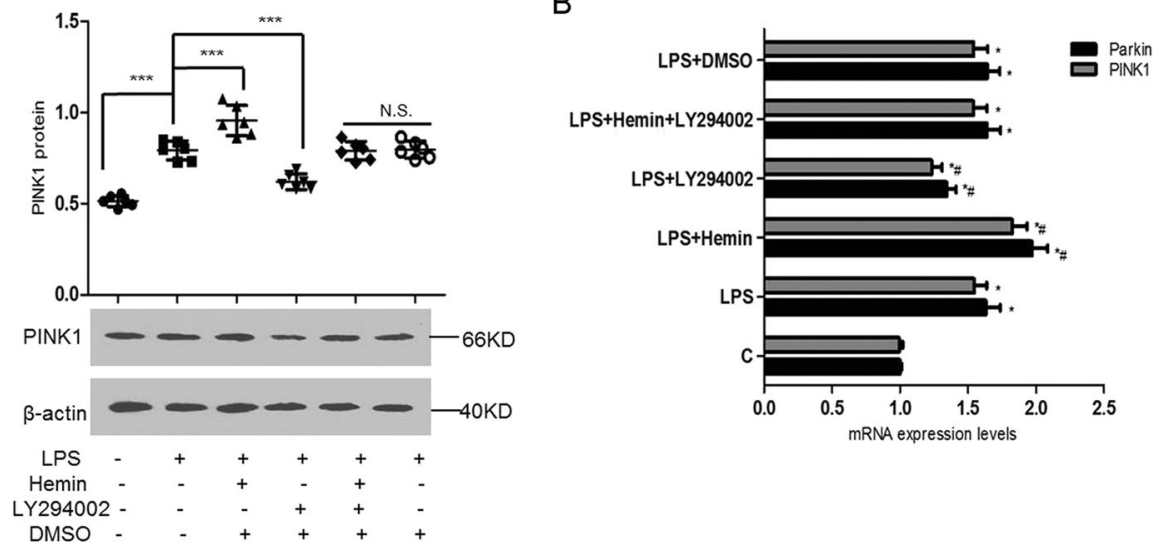

D

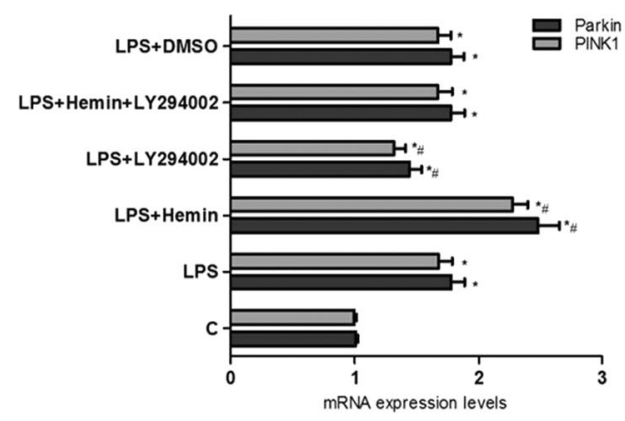

Fig. 6 Induction of HO-1 elevated dynamic changes of mitochondrial mitophagy. a, b Levels of key mediators of mitophagy, Parkin, and PINK1 were assessed by real-time PCR and western blots in RAW264.7 cells. c, $\mathbf{d}$ Representative protein or mRNA of Parkin and PINK1 expression in the lung tissues of rats. Data were presented as protein density relative to $\beta$-actin. The blots were representative of six independent experiments using one-way ANOVA corrected with
Bonferroni coefficient for multiple comparisons. Values were expressed as mean \pm s.d. $(n=6) .{ }^{*} P<0.05$ vs. control and ${ }^{\#} P<0.05$ vs. LPS-treated vehicles. Significant differences were indicated with an asterisk: ${ }^{*} P<0.05,{ }^{* *} P<0.01,{ }^{* * *} P<0.001$. LPS lipopolysaccharide, HO-1 heme oxygenase-1, PINK1 PTEN-induced putative kinase 1, NS not significant

mediated induction of $\mathrm{HO}-1$ preserved the global balance of MQC.

\section{Discussion}

In the current study, using a model of endotoxin-induced ALI in Sprague-Dawley rats or murine RAW264.7 macrophages, we demonstrated that induction of HO-1 maintained the entire process of MQC, a term collectively encompassed mitochondrial biogenesis, dynamics, and mitophagy, and further gave rise to mitigate septic lung injury. In particular, the results suggested that HO-1 induction defended against LPS-induced decline in the quality of the mitochondria, characterized by preserving the dynamic process of mitochondrial fusion/fission, promoting mitochondrial biogenesis, and facilitating mitophagy for clearance of damaged mitochondria. Most importantly, a PI3 kinase inhibitor, 

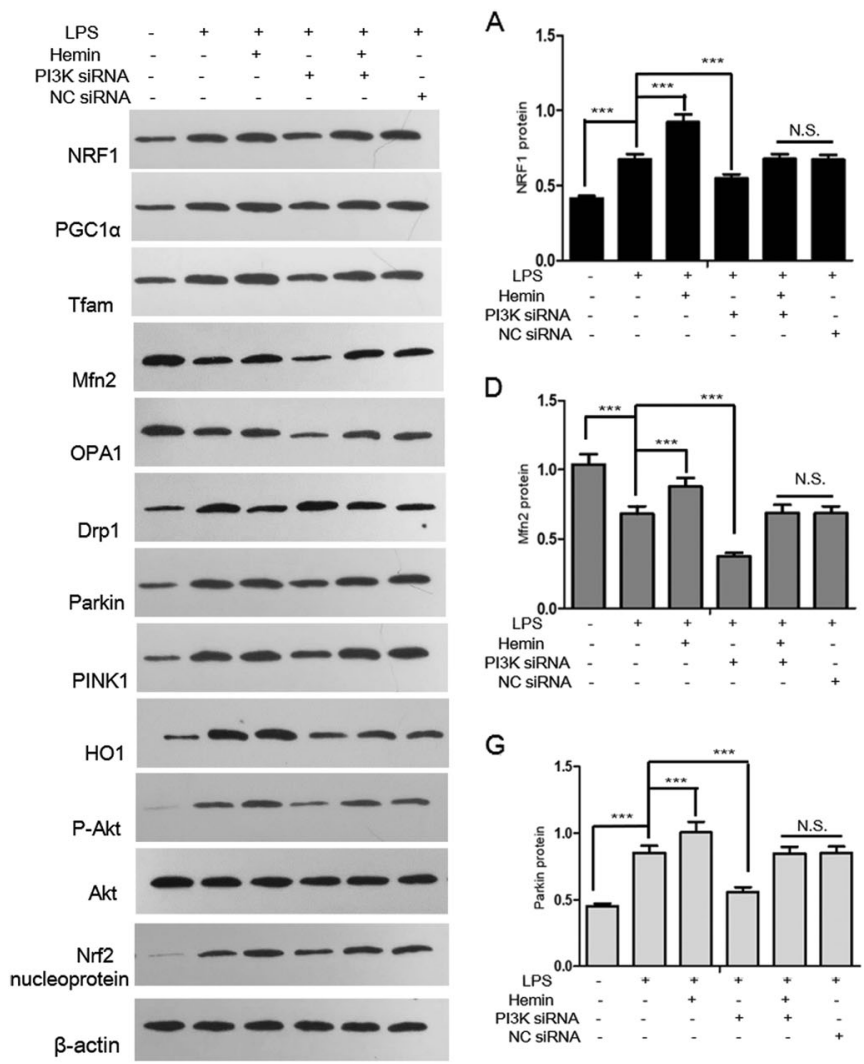

J

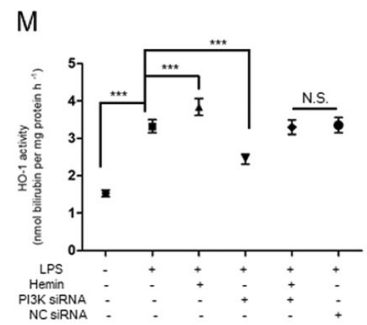

B

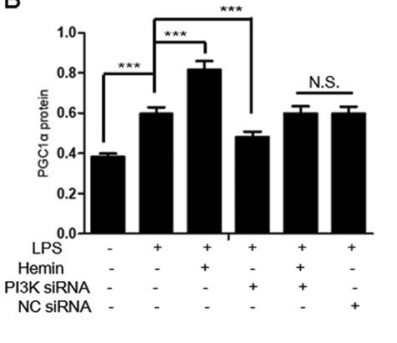

$\mathrm{E}$

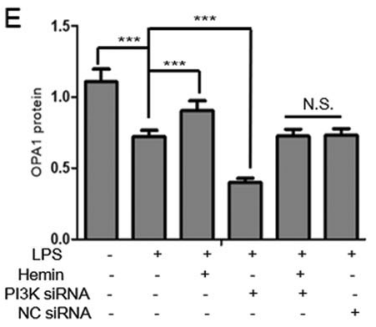

$\mathrm{H}$
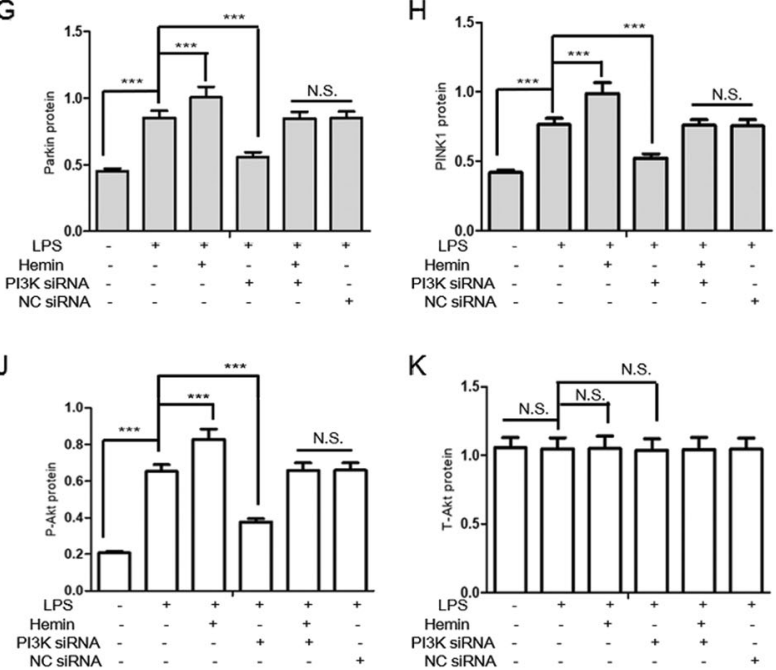

K

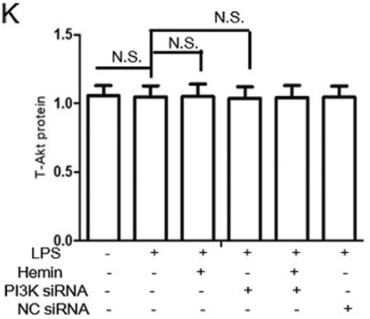

C
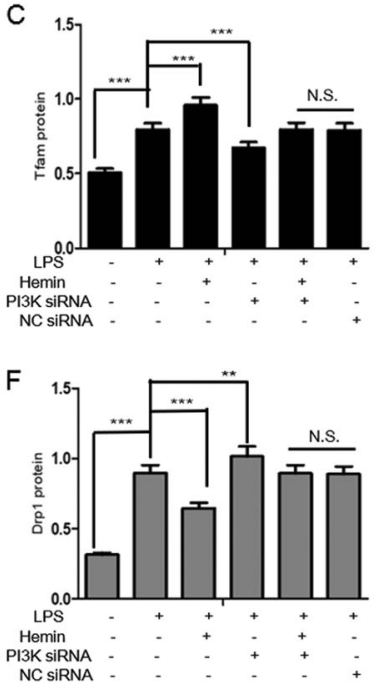

I

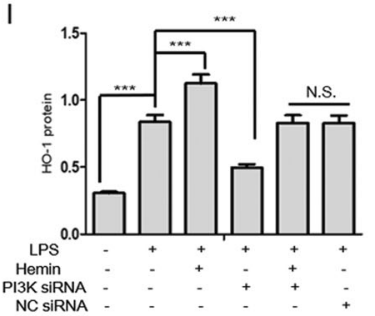

L

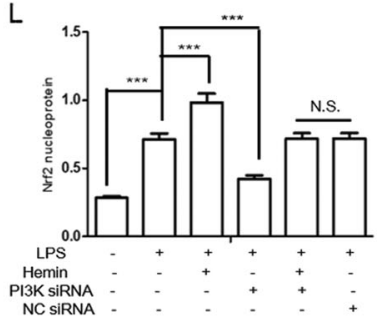

Fig. $7 \mathrm{PI} 3 \mathrm{~K} / \mathrm{Akt} / \mathrm{Nrf} 2$ pathway-mediated HO-1 induction regulated mitochondrial quality control. a-l Example bands and quantification from western blot analysis to determine the expression levels of NRF1, PGC1 $\alpha$, Tfam, Mfn2, OPA1, Drp1, Parkin, PINK1 protein necessary for mitochondrial quality control as well as HO-1, phosphor-Akt protein, and Nrf2 nucleoprotein in RAW264.2 cells. The cells transfected with negative control siRNA or PI3K siRNA and then stimulated with $1 \mu \mathrm{g} / \mathrm{ml}$ LPS with or without $20 \mu \mathrm{M}$ hemin for $24 \mathrm{~h}$. Band density was quantified relatively to $\beta$-actin. m HO-1 enzyme activity in negative control siRNA or PI3K siRNA transgenic RAW264.7 cells. All values were expressed as mean \pm s.d. obtained from six

LY294002 or PI3K knockdown with specific siRNA, abrogated HO-1 expression and abolished the salutary effects of HO-1. Collectively, we surmised that induction of HO-1 by the activation of PI3K/Akt pathway participated in preserving the MQC and alleviated septic lung injury in vitro and in vivo.

To date, up to $35-50 \%$ patients with sepsis developed into mild or severe ARDS is a compelling medical condition and starves for novel or specific therapies [38]. Oxidative mitochondrial damage secondary to septic lung independent experiments using one-way ANOVA corrected with Bonferroni coefficient for multiple comparisons. Significant differences were indicated with an asterisk: ${ }^{*} P<0.05,{ }^{* *} P<0.01,{ }^{* * *} P<$ 0.001. LPS lipopolysaccharide, Nrf2 NF-E2 related factor-2, HO-1 heme oxygenase-1, PI3K phosphatidylinositol-3-kinase, Akt protein kinase $\mathrm{B}$, NRF1 nuclear respiratory factor $1, \mathrm{PGC} 1 \alpha$ peroxisome proliferator-activated receptor $\gamma$ coactivator 1 , Tfam mitochondrial transcription factor A, Mfn2 mitofusin 2, OPA1 optic atrophy 1, Drp1 dynamin-related protein 1, PINK1 PTEN-induced putative kinase 1, NS not significant

injury has been recognized as a major cause of cell death. Recently, Carré et al. [39] reported that mitochondria dysfunction as a signature to determine the poor outcomes of septic patients. More specifically, upon to cellular stress, mitochondrial biogenesis and hyper-fusion were needed, as elongated mitochondria generated to maintain a functional network, and damaged or fragmented mitochondria were removed through the process of mitophagy [40]. In the current study, LPS-exposed RAW264.7 macrophages exhibited a notable decline in the cell viability, which was a 
commonly used model to evaluate cellular oxidative injury. Additionally, the depletion of RCR and mitochondrial membrane potential $(\Delta \Psi \mathrm{m})$, both as the most useful indicators of functional integrity of mitochondria, were found in LPS-stimulated RAW264.7 cells [41]. Concomitant with the appearance of cell viability, incubation with $1 \mu \mathrm{g} / \mathrm{ml}$ LPS resulted in the elevation of the NRF1/PGC1 $\alpha$ axis and its downstream target Tfam, boosted PINK1/Parkin-mediated mitophagy, increased the expression of Drp1 whereas Mfn2 and OPA1 levels were decreased, accompanied by swollen, fragmented mitochondria. In line with the findings in vitro, enhanced survival rates, increased lung injury scores, reduced RCR levels, depressed MnSOD activities, while raised mitochondrial biogenesis including the levels of NRF1, PGC1 $\alpha$, Tfam, elevated expression of Parkin, PINK1, as well as upregulated Drp1, yet downregulated Mfn2, OPA1 mRNA, and protein levels were observed in the lung mitochondria of rats subjected to LPS. These data indicate that it was likely that LPS exposure specifically triggered mitochondrial damage, failure to upregulate mitochondrial fusion through biogenesis, thus promoted fission of damaged mitochondria segments and then boosted mitochondrial mitophagy for their clearance, which were consistent with studies by Piantadosi et al. [42].

Importantly, HO-1 as one of arsenal of antioxidant enzymes, was indicative of cytoprotective effects against a plethora of conditions such as sepsis, hypoxia, and ischemia-reperfusion. A growing body of evidences supported the notion that HO-1 with the potently byproducts of heme catabolism, at least in part, regulated mitochondrial biogenesis or autophagy against sepsis-related multi-organ damage [43-45]. However, the close links between HO-1 induction and the global process of MQC in the context of septic lung injury were poorly understood. In regards, RAW264.7 cells or rats were pre-treated with hemin, a potent chemical HO-1 inducer and then exposed to LPS. To corroborate whether the PI3K/Akt pathway was involved in the midst of HO-1 targeted mitochondrial protection, a specific PI3K inhibitor, LY294002, or PI3K knockdown by siRNA was administrated and analyzed for the expression levels of HO-1, Akt and phosphorylation of Akt. As expected, HO-1 induction, namely, pre-incubation with hemin in LPS-stimulated macrophages resulted in loss of mitochondrial oxidative damage, as reflected in increased viability of cells, augmented levels of RCR and the $\Delta \Psi \mathrm{m}$. Likewise, there were marked concomitant increase in MnSOD activities and RCR levels, while decrease in survival rates and lung injury scores of SD rats pre-treated with hemin before LPS challenge. These findings were congruent with our previous studies [5, 6, 28]. In particular, HO-1 induction appeared to evidently upregulate the expression of Mfn2 and OPA1, downregulate the levels of Drp1, which were likely linked to the herein increase of NRF1, PGC1 $\alpha$, and Tfam-mediated mitochondrial biogenesis, as well as elevated PINK1/Parkin-mediated mitophagy. The above results were in accord with previous studies by Hull et al. [25]. In addition, changes of mitochondrial dynamics supported the observations made with TEM, which presented evidence of more numerous tubular and elongated mitochondria in hemin-pre-treated cells. In contrast, preprocessing with LY294002 inhibited the expression of HO-1 and phosphor-Akt, further deteriorated damage of MQC induced by LPS, and counteracted the protective effects offered by hemin. Moreover, pre-incubation of RAW264.7 cells with PI3K siRNA strongly blocked hemin-induced enhanced HO-1 enzyme activity as well as higher levels of HO-1, phosphor-Akt protein, and Nrf2 nucleoprotein, reversed hemin-mediated the balance of MQC, which reconfirmed that induction of HO-1 regulated MQC through the activation of PI3K/Akt/Nrf2 pathway. Combined with our previous studies [6, 37], it appeared that the PI3K/Akt pathway, acted upstream and induced nuclear translocation of Nrf2, directly worked through HO-1 on regulating MQC in the settings of septic lung injury. Similarly, the pivotal role of HO-1 in maintaining mitochondrial homeostasis to protect the lung from LPS-mediated oxidative injury has been underscored once again in vivo. By implication, PI3K/ Akt pathway-driven HO-1 induction was sufficient to ameliorate septic lung injury, primarily by preserving the overall process of mitochondrial biogenesis, dynamics, and mitophagy in vivo and in vitro. With respect to the clinical significance of the current study, novel pharmacologic agents or electroacupuncture adjuvants based on HO-1 induction are likely to be initiated to prevent sepsisassociated multiple organs dysfunction in clinic.

There were certain limitations to the current study. Firstly, there were no significant differences in the expression of total Akt protein comparing PI3K siRNA with NC siRNA-transfected macrophages, which indicated that the PI3K pathway might not be the only way of Akt activation. Therefore, to fully expound the other signaling cascades such as AMPK $\alpha$ and P38MAPK $\alpha$ pathways involved in HO-1 modulating MQC by gene knockout or gene overexpression techniques require further investigations [46]. Secondly, given that electroacupuncture at Zusanli and Neiguan acupoints facilitated lung protection against sepsis through HO-1 upregulation [47], therein, to illuminate the interplay of electroacupuncture, HO-1 system, and MQC should be the focus of future exploration, which will lay a solid foundation for its clinical applications. Thirdly, a recently research set forth that HO-1 was responsible for protective actions of bronchial epithelial cells against aggravated oxidative stress [48]. Thus, using multi-model, distinct cell types or incubation times may be more strength to fully ascertain the relationship between HO-1 system and MQC in the future. Finally, the outcome of MQC has been 
reported to reliant on the close communications with the nucleus, which play leading roles in a host of core cellular functions [49]. Thus, it is of great value to find out the endogenous effects of HO-1 on the interactions between mitochondria and other organelles and nucleus.

In conclusion, our data indicated that PI3K/Akt pathwaydependent induction of HO-1 conferred protection of the lung from ongoing oxidative injury by regulating the overall MQC network in the setting of sepsis in vitro and in vivo. Importantly, the findings described herein may be of great significance, as targeting HO-1 strategies by electroacupuncture adjuvants or pharmacologic agents to be uncovered promisingly to prevent/treat sepsis-related lung injury.

Acknowledgements This work was supported by the grants from the National Natural Science Foundation of China (\#81772106), Key project of Tianjin Natural Science Foundation (18JCZDJC35400) and Youth project of Tianjin Natural Science Foundation (18JCQNJC11000).

\section{Compliance with ethical standards}

Conflict of interest The authors declare that they have no conflict of interest.

Publisher's note: Springer Nature remains neutral with regard to jurisdictional claims in published maps and institutional affiliations.

\section{References}

1. Otterbein LE, Foresti R, Motterlini R. Heme oxygenase-1 and carbon monoxide in the heart: the balancing act between danger signaling and pro-survival. Circ Res. 2016;118:1940-59.

2. Chen $\mathrm{X}, \mathrm{Wu} \mathrm{S}$, Tang L, et al. Mesenchymal stem cells overexpressing heme oxygenase-1 ameliorate lipopolysaccharideinduced acute lung injury in rats. J Cell Physiol. 2019;234:7301-19.

3. MacGarvey NC, Suliman HB, Bartz RR, et al. Activation of mitochondrial biogenesis by heme oxygenase-1-mediated NF-E2related factor-2 induction rescues mice from lethal Staphylococcus aureus sepsis. Am J Respir Crit Care Med. 2012;185:851-6.

4. Fan E, Brodie D, Slutsky AS. Acute respiratory distress syndrome advances in diagnosis and treatment. JAMA. 2018;319:698-710.

5. Yu JB, Shi J, Wang D, et al. Heme oxygenase-1/carbon monoxide regulated mitochondrial dynamic equilibrium contributes to the attenuation of endotoxin-induced acute lung injury in rats and in lipopolysaccharide-activated RAW264.7 cells. Anesthesiology. 2016;125:1190-201.

6. Yu JB, Wang Y, Li Z, et al. Effect of heme oxygenase-1 on mitofusion-1 protein in LPS-induced ALI/ARDS in rats. Sci Rep. 2016;6:36530.

7. Murphy MP, Hartley RC. Mitochondria as a therapeutic target for common pathologies. Nat Rev Drug Discov. 2018. https://doi.org/ 10.1038/nrd.2018.174

8. Youle RJ, van der Bliek AM. Mitochondrial fission, fusion and stress. Science. 2012;337:1062-5.

9. Zhang CS, Lin SC. AMPK promotes autophagy by facilitating mitochondrial fission. Cell Metab. 2016;23:399-401.

10. Braun RJ, Westermann B. With the help of MOM: mitochondrial contributions to cellular quality control. Trends Cell Biol. 2017; 27:441-52.
11. Picca A, Mankowski RT, Burman JL, et al. Mitochondrial quality control mechanisms as molecular targets in cardiac ageing. Nat Rev Cardiol. 2018;15:543-54.

12. Park JS, Choi HS, Yim SY, et al. Heme oxygenase-1 protects the liver from septic injury by modulating TLR4-mediated mitochondrial quality control in mice. Shock. 2018;50:209-18.

13. Piantadosi CA, Suliman HB. Mitochondrial dysfunction in lung pathogenesis. Annu Rev Physiol. 2017;79:495-515.

14. Chang AL, Ulrich A, Suliman HB, et al. Redox regulation of mitophagy in the lung during murine Staphylococcus aureus sepsis. Free Radic Biol Med. 2015;78:179-89.

15. Cantley L. The phosphoinositide 3-kinase pathway. Science. 2002;296:1655-7.

16. Hu H, Juvekar A, Lyssiotis CA, et al. Phosphoinositide 3-kinase regulates glycolysis through mobilization of aldolase from the actin cytoskeleton. Cell. 2016;164:433-46.

17. Pei L, Kong Y, Shao C, et al. Heme oxygenase-1 induction mediates chemoresistance of breast cancer cells to pharmorubicin by promoting autophagy via PI3K/Akt pathway. J Cell Mol Med. 2018;22:1-11.

18. Xiao Q, Piao R, Wang $\mathrm{H}$, et al. Orientin-mediated Nrf2/HO1 signal alleviates $\mathrm{HO}$-induced oxidative damage via induction of $\mathrm{JNK}$ and PI3K/AKT activation. Int $\mathrm{J}$ Biol Macromol. 2018;118:747-55

19. Kim H, Kim SR, Je J, et al. The proximal tubular $\alpha 7$ nicotinic acetylcholine receptor attenuates ischemic acute kidney injury through Akt/PKC signaling-mediated HO-1 induction. Exp Mol Med. 2018;50:40.

20. Wang Y, Xu Y, Zhang P, et al. Smiglaside A ameliorates LPSinduced acute lung injury by modulating macrophage polarization via AMPK-PPAR $\gamma$ pathway. Biochem Pharmacol. 2018;156:1-47.

21. $\mathrm{Xu} \mathrm{L}, \mathrm{Zhao} \mathrm{P}, \mathrm{Xu} \mathrm{Y}$, et al. Tim-4 protects mice against lipopolysaccharide-induced endotoxic shock by suppressing the NF-кB signaling pathway. Lab Invest. 2016;96:1189-97.

22. Suliman HB, Keenan JE, Piantadosi CA. Mitochondrial qualitycontrol dysregulation in conditional HO-1-/- mice. JCI Insight. 2017;2:e89676

23. Zheng X, Wu J, Shao Q, et al. Apoptosis of THP-1 macrophages induced by pseudohypericin-mediated sonodynamic therapy through the mitochondria-caspase pathway. Cell Physiol Biochem. 2016;38:545-57.

24. Ma X, You X, Zeng Y, et al. Mycoplasma fermentans MALP-2 induces heme oxygenase-1 expression via mitogen-activated protein kinases and Nrf2 pathways to modulate cyclooxygenase 2 expression in human monocytes. Clin Vaccine Immunol. 2013;20:827-34.

25. Hull TD, Boddu R, Guo L, et al. Heme oxygenase-1 regulates mitochondrial quality control in the heart. JCI Insight. 2016;1: e85817.

26. Wen J, Grenz A, Zhang Y, et al. A2B adenosine receptor contributes to penile erection via PI3K/AKT signaling cascademediated eNOS activation. FASEB J. 2011;25:2823-30.

27. Schmittgen TD, Livak KJ. Analyzing real-time PCR data by the comparative C(T) method. Nat Protoc. 2008;3:1101-8.

28. Shi J, Yu JB, Liu W, et al. Carbon monoxide alleviates lipopolysaccharide-induced oxidative stress injury through suppressing the expression of Fis1 in NR8383 cells. Exp Cell Res. 2016;349:162-7.

29. Wang XL, Pan LL, Long E, et al. Endogenous hydrogen sulfide ameliorates NOX4 induced oxidative stress in LPS-stimulated macrophages and mice. Cell Physiol Biochem. 2018;47:458-74.

30. Vettorazzi S, Bode C, Dejager L, et al. Glucocorticoids limit acute lung inflammation in concert with inflammatory stimuli by induction of SphK1. Nat Commun. 2015;6:7796.

31. Sommer SP, Sommer S, Sinha B, et al. Ischemia-reperfusion injury-induced pulmonary mitochondrial damage. J Heart Lung Transplant. 2011;30:811-8. 
32. Jacoby RP, Millar AH, Taylor NL. Assessment of respiration in isolated plant mitochondria using Clark-type electrodes. Methods Mol Biol. 2015;1305:165-85.

33. Brealey D, Brand M, Hargreaves I, et al. Association between mitochondrial dysfunction and severity and outcome of septic shock. Lancet. 2002;360:219-23.

34. Hart PC, Mao M, de Abreu AL, et al. MnSOD upregulation sustains the Warburg effect via mitochondrial ROS and AMPKdependent signaling in cancer. Nat Commun. 2015;6:6053.

35. Ni HM, Williams JA, Ding WX. Mitochondrial dynamics and mitochondrial quality control. Redox Biol. 2015;4:6-13.

36. Piquereau J, Godin R, Deschênes $S$, et al. Protective role of PARK2/Parkin in sepsis-induced cardiac contractile and mitochondrial dysfunction. Autophagy. 2013;9:1837-51.

37. Yu JB, Shi J, Zhang Y, et al. Electroacupuncture ameliorates acute renal injury in lipopolysaccharide-stimulated rabbits via induction of $\mathrm{HO}-1$ through the PI3K/Akt/Nrf2 pathways. PLoS ONE. 2015;10:e0141622.

38. Cecconi M, Evans L, Levy M, et al. Sepsis and septic shock. Lancet. 2018;392:75-87.

39. Carré JE, Orban JC, Re L, et al. Survival in critical illness is associated with early activation of mitochondrial biogenesis. Am J Respir Crit Care Med. 2010;182:745-51.

40. Wang Y, Subramanian M, Yurdagul A, et al. Mitochondrial fission promotes the continued clearance of apoptotic cells by macrophages. Cell. 2017;171:331-45.

41. Brand MD, Nicholls DG. Assessing mitochondrial dysfunction in cells. Biochem J. 2011;435:297-312.
42. Piantadosi CA, Carraway MS, Babiker A, et al. Heme oxygenase1 regulated cardiac mitochondrial biogenesis via Nrf2-mediated transcriptional control of nuclear respiratory factor-1. Circ Res. 2008;103:1232-40.

43. Li XY, Zhang Y, Yu JB, et al. Activation of protein kinase C- $\alpha /$ heme oxygenase-1 signaling pathway improves mitochondrial dynamics in lipopolysaccharide-activated NR8383 cells. Exp Ther Med. 2018;16:1529-37.

44. Rayamajhi N, Kim SK, Go H, et al. Quercetin induces mitochondrial biogenesis through activation of HO-1 in HepG2 cells. Oxid Med Cell Longev. 2013;2013:154279.

45. Carchman EH, Rao J, Loughran PA, et al. Heme oxygenase-1mediated autophagy protects against hepatocyte cell death and hepatic injury from infection/sepsis in mice. Hepatology. 2011;53: 2053-62.

46. Cho RL, Lin WN, Wang CY, et al. Heme oxygenase-1 induction by rosiglitazone via PKC $\alpha / \mathrm{AMPK} \alpha / \mathrm{p} 38 \mathrm{MAPK} \alpha / \mathrm{SIRT} 1 / \mathrm{PPAR} \gamma$ pathway suppresses lipopolysaccharide-mediated pulmonary inflammation. Biochem Pharmacol. 2018;148:222-37.

47. Yu JB, Shi J, Gong LR, et al. Role of Nrf2/ARE pathway in protective effect of electroacupuncture against endotoxic shockinduced acute lung injury in rabbits. PLoS ONE. 2014;9: e104924.

48. Abdalla MY, Hoke T, Seravalli J, et al. Pseudomonas quinolone signal induces oxidative stress and inhibits heme oxygenase-1 expression in lung epithelial cells. Infect Immun. 2017;85:1-39.

49. Quirós PM, Mottis A, Auwerx J. Mitonuclear communication in homeostasis and stress. Nat Rev Mol Cell Biol. 2016;17:213-26. 